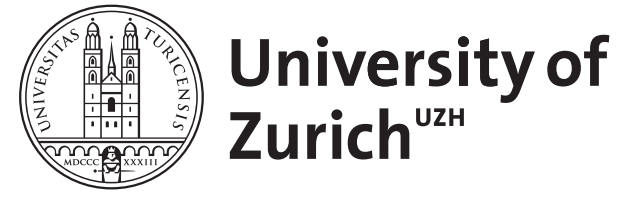

Zurich Open Repository and Archive

University of Zurich

University Library

Strickhofstrasse 39

CH-8057 Zurich

www.zora.uzh.ch

Year: 2012

\title{
Wechselwirkung von Medikamenten mit der Grunderkrankung - Wenn Medikamente Krankheiten verschlechtern
}

\author{
Corti, Natascia ; Taegtmeyer, Anne ; Curkovic, Ivanka
}

DOI: https://doi.org/10.1024/1661-8157/a000877

Posted at the Zurich Open Repository and Archive, University of Zurich ZORA URL: https://doi.org/10.5167/uzh-71663

Journal Article

Accepted Version

Originally published at:

Corti, Natascia; Taegtmeyer, Anne; Curkovic, Ivanka (2012). Wechselwirkung von Medikamenten mit der Grunderkrankung - Wenn Medikamente Krankheiten verschlechtern. Praxis, 101:363-370.

DOI: https://doi.org/10.1024/1661-8157/a000877 
DOI 10.1024/1661-8157/a000877

((Rubrik CME))

\section{Wechselwirkung von Medikamenten mit der Grunderkrankung - Wenn Medikamente Krankheiten verschlechtern}

\section{Im Artikel verwendete Abkürzungen}

ACE Angiotensin-konvertierendes Enzym

AT-II Angiotensin-II-Rezeptor

CDAI Crohn's Disease Activity Index

G6PD Glucose-6-Phosphat-Dehydrogenase

NSAR: Nicht-steroidale Antirheumatika

NYHA New York Heart Association

\section{Einführung ((Ü1))}

Unerwünschte Arzneimittelwirkungen können einerseits Folge von Wechselwirkungen zwischen den einzelnen Arzneimitteln sein. Dabei führen Arzneimittelwechselwirkungen zu einer unbeabsichtigten Wirkungsverstärkung oder Wirkungsabschwächung. Andererseits können Arzneimittel auch mit den bestehenden Erkrankungen interagieren, was zur ungewollten Exazerbation von chronischen Grunderkrankungen führen kann. Oft ist die Verschreibung eines Arzneimittels gerade bei Polymorbiden und somit insbesondere älteren Patienten eine Gratwanderung zwischen Nutzen und gleichzeitigem Abwägen des Risikos einer unerwünschten Arzneimittelwirkung. In retrospektiven Analysen konnte in $20-50 \%$ der betagten Patienten eine in Bezug auf die Grunderkrankung ungeeignete Medikation identifiziert werden, die potenziell zu unerwünschten Arzneimittelwirkungen im Sinne einer Exazerbation der Grunderkrankungen hätte führen können [1]. Unter den am häufigsten angeschuldigten Medikamenten wurden unter anderem die nicht-steroidalen Antirheumatika (NSAR) inklusive Thrombozytenaggregations-Hemmer und kardiovaskuläre Medikamente identifiziert. Durch konsequenten Einbezug der «Kontraindikationen» und der «Warnhinweise und Vorsichtsmassnahmen» in den jeweiligen Produkteinformationen bei der Arzneimittelverschreibung, könnte ein Grossteil dieser Probleme vermieden werden. Auch existieren Listen ungeeigneter Medikamente beim betagten Patienten (De Beers [2], www.priscus.net), die das Bewusstsein für das Interaktionsrisiko von Arzneimitteln mit der zugrundeliegenden Erkrankung insbesondere beim betagten Patienten erhöhen sollen. Dennoch zeigt die tägliche Verschreibungspraxis, dass ebendiese ungeeigneten respektive sogar kontraindizierten Substanzen immer wieder verabreicht werden - mit den entsprechenden Konsequenzen.

Etwas seltener ist man als Verschreibender mit Patienten konfrontiert, die unter einer angeborenen Erkrankung leiden, bei welcher eine Wechselwirkung bestimmter Medikamente mit der Grunderkrankung potenziell schwerwiegende Konsequenzen haben kann. Dazu gehören unter anderem hepatische Porphyrien, Myasthenia gravis, Favismus oder das angeborene Long-QT-Syndrom. Vor einer medikamentösen Therapie sollten in diesen Fällen die entsprechenden (im Internet) verfügbaren Listen (Abb. 1) konsultiert und die absolut kontraindizierten Substanzen gemieden werden. An durch Patienten selbst eingesetzte freiverkäufliche Medikamente wie z.B. NSAR, Antihistaminika oder Antiemetika ist insbesondere bei betagten Patienten, die sich wegen einer Exazerbation oder Verschlechterung ihrer chronischen Krankheit vorstellen, immer zu denken und entsprechend nachzufragen. 
Abbildung 1: Links zu Listen kontraindizierter Medikamente

\begin{tabular}{ll}
\hline Kondition & Medikationsliste aufrufbar bei \\
\hline Porphyrie & http://www.drugs-porphyria.org/ \\
& http://www.porphyria.ch/ \\
Myasthenie & http://www.myasthenia-gravis.de/infos/vermeiden.htm \\
G6PD-Mangel & http://www.g6pd.org/favism/english/index.mvc \\
& http://www.favismus.de/ \\
Long-QT-Syndrom & http://www.azcert.org/ \\
Brugada-Syndrom & http://brugadadrugs.org/ \\
\hline
\end{tabular}

In diesem CME wird aufgrund der Häufigkeit ihres Einsatzes und der teilweise rezeptfreien Verfügbarkeit auf Schmerzmittel, Antiemetika und Antihistaminika fokussiert. Abbildung 2 stellt eine Übersicht von Grunderkrankungen dar, bei denen die genannten Arzneimittel ungeeignet sind, mit den entsprechenden Beispielen für mögliche Alternativen. Diese Liste ist nicht abschliessend. Auch bei den Alternativ-Medikamenten sollte jeweils die Produkteinformation in Hinsicht auf Risiken und Kontraindikationen konsultiert werden.

Abbildung 2: Wechselwirkung von Analgetika, Antiemetika und Antihistaminika mit der Grunderkrankung sowie alternative Therapien

\begin{tabular}{|c|c|c|c|}
\hline Gruppe & $\begin{array}{l}\text { Medikamentenklasse oder } \\
\text { Medikament }\end{array}$ & $\begin{array}{l}\text { Grunderkrankung die durch } \\
\text { Einnahme des Medikamentes } \\
\text { exazerbieren kann }\end{array}$ & Mögliche Alternativen \\
\hline Analgetika & NSAR und Coxibe & $\begin{array}{l}\text { COPD (bei reversibler Obstruktion) } \\
\text { Arterielle Hypertonie } \\
\text { Herzinsuffizienz } \\
\text { Koronare Herzkrankheit } \\
\text { Chronisch-entzündliche } \\
\text { Darmerkrankung (siehe } \\
\text { Fallbeispiel) } \\
\text { Niereninsuffizienz }\end{array}$ & $\begin{array}{l}\text { Paracetamol } \\
\text { Metamizol (Novalgin } \AA)\end{array}$ \\
\hline & Metamizol (Novalgin $®)$, NSAR & Asthma bronchiale & $\begin{array}{l}\text { Paracetamol, Opioide } \\
\text { (bei akutem Asthma } \\
\text { allerdings wegen } \\
\text { Atemdepressionsgefahr } \\
\text { kontraindiziert) }\end{array}$ \\
\hline & $\begin{array}{l}\text { Tramadol (Tramal®), } \\
\text { Hydromorphon (Palladon®), } \\
\text { Pethidin }\end{array}$ & Epilepsie & Morphin, Fentanyl \\
\hline
\end{tabular}




\begin{tabular}{|c|c|c|c|}
\hline & Trizyklische Antidepressiva, & $\begin{array}{l}\text { Kognitive Einschränkung, Demenz } \\
\text { Miktionsstörung bei z.B. } \\
\text { Prostatahypertrophie mit } \\
\text { Restharnbildung und } \\
\text { Blasenhalsobstruktion, } \\
\text { Engwinkelglaukom, } \\
\text { Epilepsie }\end{array}$ & $\begin{array}{l}\text { Gabapentin } \\
\text { (Neurontin®) } \\
\text { Pregabalin (Lyrica®) }\end{array}$ \\
\hline \multirow[t]{2}{*}{ Antiemetika } & Metoclopramid (Paspertin $®)$ & Morbus Parkinson & $\begin{array}{l}\text { Domperidon } \\
{\text { (Motilium } \AA)^{* *}}^{*}\end{array}$ \\
\hline & Meclozin (Itinerol $\circledast \mathrm{B}_{6}$ ) & $\begin{array}{l}\text { Engwinkelglaukom, } \\
\text { Prostatahypertrophie mit } \\
\text { Restharnbildung und } \\
\text { Blasenhalsobstruktion }\end{array}$ & $\begin{array}{l}\text { Domperidon } \\
(\text { Motilium } \AA)^{\star *} \\
\text { Metoclopramid } \\
\text { (Paspertin } \circledast)\end{array}$ \\
\hline Antihistaminika & Clemastin (Tavegy|®) & $\begin{array}{l}\text { Engwinkelglaukom, } \\
\text { Prostatahypertrophie mit } \\
\text { Restharnbildung und } \\
\text { Blasenhalsobstruktion, } \\
\text { Kognitive Einschränkung, } \\
\text { Demenz }\end{array}$ & $\begin{array}{l}\text { z.B. Levocetirizin } \\
\text { (Xyzal®), Fexofenadin } \\
\text { (Telfast®) u.a. }\end{array}$ \\
\hline
\end{tabular}

** Bei Long-QT-Syndrom kontraindiziert 
Im Fallbeispiel wird die Assoziation zwischen der Einnahme von NSAR und Exazerbation eines M. Crohn vorgestellt. Die Einnahme von NSAR bei Patienten mit Colitis ulcerosa ist ebenfalls kontraindiziert. Der Pathomechanismus bezieht sich auf die Reduktion der Prostaglandinsynthese, die unter anderem zu einer Einschränkung der Mikrozirkulation der intestinalen Mukosa führt.

Die Hemmung der Prostaglandinsynthese durch NSAR ist auch für die Asthma-Exazerbation verantwortlich. Arachidonsäure wird in Anwesenheit von Cyclooxygenase-Hemmern vermehrt zu Leukotrienen verstoffwechselt. Leukotriene können eine Bronchokonstriktion, eine vermehrte Schleimproduktion sowie die Migration von inflammatorischen Zellen in die Bronchi auslösen.

Metamizol (Novalgin $\circledast$ ) wird aufgrund eines erhöhten Risikos für anaphylaktische Reaktionen bei Patienten mit Asthma ebenfalls nicht empfohlen.

NSAR verursachen durch die Prostaglandinsynthese-Hemmung auch eine systemische Vasokonstriktion, was zu einer Erhöhung des arteriellen Blutdruckes führen kann. NSAR können zudem die Wirkung von ACE-Hemmern und AT-II-Blockern abschwächen, weshalb diese Schmerzmittel bei Patienten mit arterieller Hypertonie nur mit Vorsicht eingesetzt werden sollten. Durch eine Reduktion der Nierenperfusion kann es zusätzlich zu einer Nierenfunktionsstörung kommen, die eine Wasserretention und Hyponatriämie verursacht. Formal stellt eine New York Heart Association (NYHA)-Klasse III-IV bei einer Herzinsuffizienz gemäss der schweizerischen Fachinformation eine absolute Kontraindikation zur Ibuprofen- oder Diclofenac-Therapie dar. Aufgrund von kardiovaskulären unerwünschten Arzneimittelwirkungen sind auch Coxibe (z.B. Celebrex®) bei Patienten mit Herzinsuffizienz der NYHA-Klasse II-IV, aber auch mit koronarer Herzkrankheit oder zerebrovaskulären Erkrankungen kontraindiziert [3].

\section{Opioide ((Ü2))}

Die Einnahme des Opioides Tramadol ist mit Krampfanfällen assoziiert. Ein erhöhtes Risiko besteht bei der Verabreichung von hohen Dosen, insbesondere beim Überschreiten der empfohlenen maximalen Tagesdosis von $400 \mathrm{mg}$ und in Kombination mit anderen Krampfschwelle-senkenden Substanzen. Tramadol ${ }^{\circledR}$ darf gemäss Fachinformation bei medikamentös ungenügend kontrollierter Epilepsie nicht angewendet werden. Weitere Beispiele für Opioide, die die Krampfneigung erhöhen sind Hydromorphon (Palladon $\circledast$ ) und Pethidin, weshalb diese bei Patienten mit Neigung zu Krampfanfällen nicht eingesetzt werden sollten und alternative Opiate wie z.B. Morphin oder Fentanyl vorgezogen werden sollte. Bei chronisch obstruktiver Lungenerkrankung bergen alle Opioide ein erhöhtes Risiko für Atemdepression.

Der Einsatz von trizyklischen Antidepressiva wie z.B. Amitriptylin (Saroten®) ist bei gewissen Patienten mit chronischen, insbesondere auch neuropathischen Schmerzen, die sich gegenüber der üblichen analgetischen Behandlung als resistent erwiesen haben, eine mögliche Therapiealternative. Trizyklische Antidepressiva besitzen zusätzlich zur Hemmung der Serotonin- und Noradrenalin-Wiederaufnahme einen ausgeprägten anticholinergen Effekt, weshalb bereits bei therapeutischen Dosen, insbesondere bei älteren Menschen, anticholinerge Effekte wie Mundtrockenheit, Verschwommensehen (durch Mydriase), Verwirtheit und Urinretention auftreten können. Die Mydriase entsteht durch die nicht-antagonisierte Wirkung des Dilatator pupillae-Muskels mit der Folge, dass Augenwasser suboptimal abfliessen kann und es zu einer Erhöhung des intraokulären Druckes kommen kann. Das unbehandelte Engwinkelglaukom stellt deshalb eine absolute und das behandelte Engwinkelglaukom eine relative Kontraindikation zur Amitriptylin-Therapie dar. Ähnlich ist es bei der Blasenhalsobstruktion (z.B. durch Prostatahypertrophie), bei welcher der anticholinerge Effekt die Kontraktion des 
parasympathisch innervierten Detrusor-Muskels verhindert, was zu einer Harnretention führen kann. Akuter Harnverhalt stellt deshalb eine absolute Kontraindikation und Prostatahypertrophie eine relative Kontraindikation für eine Therapie mit Amitriptylin dar. Anticholinerg wirksame Substanzen erhöhen insbesondere beim alten Menschen das Risiko von Verwirrtheit und Delir, weshalb Trizyklika beim Betagten mit Vorsicht eingesetzt werden sollten. Von den Antidepressiva haben die Trizyklika das höchste Risko für eine QTc-Zeit-Verlängerung [3].

\section{Antiemetika ((Ü1))}

Metoclopramid ist ein zentral wirksamer Dopamin-Antagonist und kann daher ein vorbestehendes Parkinsonsyndrom, dem ursächlich ein Dopamin-Mangel in der Substantia nigra zugrundeliegt, exazerbieren. Alternativ kann der nur peripher wirksame Dopamin-Antagonist Domperidon (Motilium®) bei Patienten mit Parkinsonsyndrom eingesetzt werden. Domperidon ist allerdings mit QTc-Zeit-Verlängerung assoziiert und sollte nur bei normaler QTc-Zeit und in Abwesenheit von anderen Medikamenten, die die QTc-Zeit verlängern, benutzt werden. Ein erhöhtes Risiko besteht insbesondere in Kombination mit Substanzen, die den DomperidonMetabolismus hemmen (CYP3A4-Hemmer) und selbst die QTc-Zeit verlängern können. Dazu gehören die Azolantimykotika (z.B. Itraconazol, Ketoconazol), Makrolidantibiotika (z.B. Clarithromycin) aber auch das Antiarrhythmikum Amiodaron, weshalb die gleichzeitige Verabreichung von Domperidon mit diesen Medikamenten kontraindiziert ist. Auch 5HT3-Antagonisten wie Ondansetron und Tropisetron haben, insbesondere bei hoher Dosierung und intravenöser Verabreichung, das Potenzial, die QTc-Zeit zu verlängern. Bei entsprechender Anamnese und QTc-Zeit-verlängernder Komedikation ist deshalb Vorsicht geboten.

Das Antihistaminikum Itinerol $B_{6}{ }^{\circledR}$ (Meclozin, Pyridoxin, Koffein) ist ein bei Übelkeit sehr häufig eingesetztes Antiemetikum, das als Antihistaminikum ebenfalls eine anticholinerge Wirkung besitzt und deshalb eine relative Kontraindikation für Patienten mit Engwinkelglaukom und Prostatahypertrophie darstellt [4].

Antihistaminika ((Ü1))

Unter den Antihistaminika zeigen die älteren Erstgenerationspräparate wie Diphenhydramin (Benocten®), Clemastin (Tavegyl®) oder Doxylamin (Sanalepsi®) zusätzliche anticholinerge Wirkungen. Sie passieren im Gegensatz zu den neueren Antihistaminika der zweiten und dritten Generation die Bluthirnschranke und üben so auch eine zentrale anticholinerge und sedierende Wirkung aus. Die Atropin-artigen Effekte und die ausgeprägte Sedation haben dazu geführt, dass die älteren Antihistaminika zur Behandlung von Allergien kaum mehr eine Rolle mehr spielen. Allerdings sind sie in der Selbstmedikation als rezeptfreie und vermeintlich harmlose Schlafmittel noch von Bedeutung und bergen bei unkritischer Anwendung Risiken [5]. Mundtrockenheit, Harnretetion, eine Erhöhung des Augeninnendruckes, Verwirrtheitszustände und Obstipation sind typische, besonders bei älteren Patienten beobachtete [6], anticholinerge Wirkungen, weshalb die Präparate bei entsprechenden Vorerkrankungen (Engwinkelglaukom, Prostatahypertrophie mit Blasenhalobstruktion, kognitive Einschränkung) zu vermeiden sind. Hinzu kommt das arrhythmogene Potenzial (QTc-Zeit-Verlängerung) älterer Antihistaminika wie Diphenhydramin bei hoher Dosierung. Die meisten Zweit- und DrittgenerationsAntihistaminika zeigen hingegen keine klinisch relevanten anticholinergen und kaum sedierende Effekte, auch sind sie bezüglich kardialer Toxizität unproblematisch. Beispiele sind Fexofenadin (Telfast $\left.{ }^{\circledR}\right)$, Levocetirizin (Xyzal®), Loratadin (Claritine®) und Desloratadin (Aerius®) [5]. 


\section{Fallbericht zu Wechselwirkung von Medikamenten mit der Grunderkrankung}

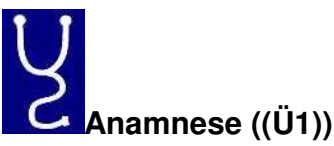

Kommentar ((Ü1))

Ein 62-jähriger Mann mit bekanntem Morbus Crohn seit fast 40 Jahren erhielt im Februar 2010 wegen einer fortgeschrittenen Arthrose des rechten oberen Sprunggelenkes ein arthroskopisches Gelenkdebridement mit partieller Synovektomie. Unter Dauertherapie mit Humira ${ }^{\circledR} 40$ mg subkutan alle 14 Tagen (Adalimumab Anti-Tumornekrosefaktor alpha) seit August 2007 und systemischen Kortikosteroiden (Prednison 12,5 mg/Tag) war er klinisch in Remission. Als Nebendiagnosen bestanden eine arterielle Hypertonie, die mit einem ACEHemmer behandelt war und eine leichte Ostopenie.

Zur postoperativen Analgesie erhielt er Mephadolor® 500 Neo (Mefenaminsäure) 500 mg dreimal täglich. Er wurde am zweiten postoperativen Tag nach Hause entlassen. Die Therapie mit Mefenaminsäure wurde zuhause weitergeführt. Am Folgetag trat ein schwerer M. Crohn-Schub mit Koliken und nicht-blutigen Durchfällen bis zu zehnmal täglich ein. Weil er sich die Ursache des Schubes nicht erklären konnte, las er sorgfältig die Mephadolor® $500 \mathrm{Neo}$

Packungsbeilage. Er stellte fest, dass Mephadolor® 500

Neo bei Morbus Crohn nicht eingenommen werden darf. Er brach die Therapie selbstständig ab und erhöhte die Prednisondosis auf $50 \mathrm{mg}$ täglich während zwei Tagen. Die Symptomatik verbesserte sich darunter rasch, so dass er anschliessend das Prednison alle zwei bis fünf Tage bis zu einer Erhaltungsdosis von $17,5 \mathrm{mg}$ pro Tag reduzierte. Einen Monat später stellte er sich bei seinem behandelnden Gastroenterologen zur Verlaufskontrolle vor. Dem Patienten wurde eine Liste von zu vermeidenden Analgetika ausgehändigt (siehe unter «Zukünftige Massnahmen»).

\section{d}

Befunde des Patienten 4 Wochen später ((Ü1))

\section{Kommentar ((Ü1))}

\section{Status ((Ü3))}

Guter Allgemein- und Ernährungszustand, afebril, normokard und normoton. Abdomen weich, leichte Der Status deutete auf eine klinische Remission hin.

Der Patient hat innerhalb von zwei Tagen nach Einnahme von Mefenaminsäure einen M. CrohnSchub entwickelt. In der Fachinformation zu Mephadolor® $500 \mathrm{Neo}$ (Mefenaminsäure) sind chronisch-entzündliche Darmerkrankungen als Kontraindikation aufgeführt.

Die jahrzehntelange Krankheitserfahrung ging mit einer Selbsttherapie des akuten Schubes einher, so dass zum Zeitpunkt der Exazerbation keine klinische Kontrolle durch den behandelnden Gastroenterologen erfolgen konnte. generalisierte Druckdolenz, mittel bis lebhafte Darmgeräusche über allen vier Quadranten. Keine 


\begin{tabular}{|c|c|}
\hline Resistenzen, keine Organomegalie. & \\
\hline 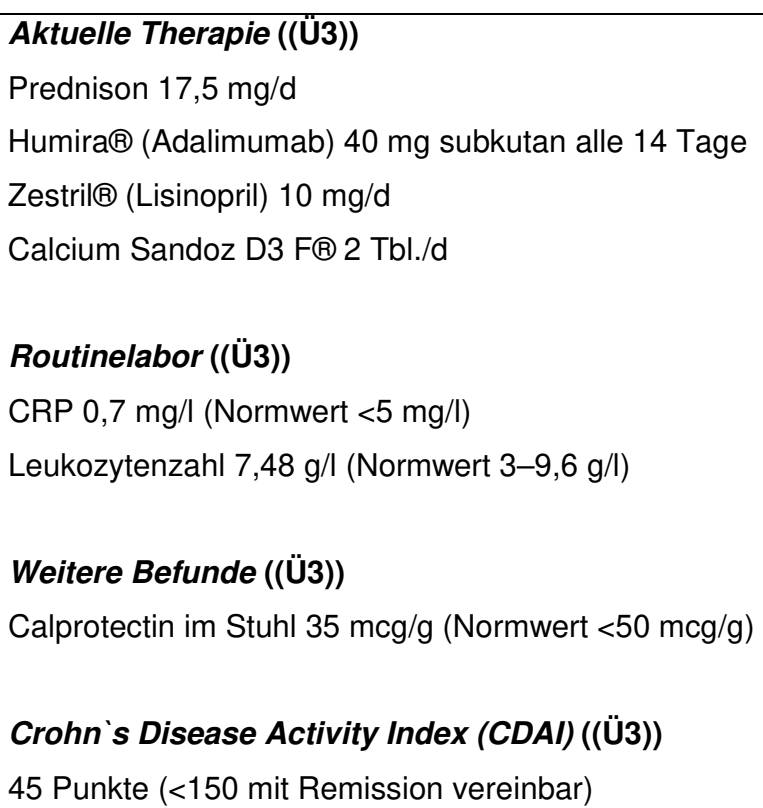 & $\begin{array}{l}\text { Die Laborbefunde sowie der CDAI Score deuteten } \\
\text { auf eine klinische Remission hin. Die vom } \\
\text { Patienten vernünftig und rechtzeitig eingeleiteten } \\
\text { Massnahmen konnten möglicherweise einen } \\
\text { schweren Verlauf verhindern. }\end{array}$ \\
\hline
\end{tabular}

45 Punkte (<150 mit Remission vereinbar)

Zukünftige Massnahmen ((Ü1))

Patienten mit chronisch-entzündlichen Darmerkrankungen können folgende Analgetika einnehmen (in Klammern jeweils Beispiele der Handelsnamen):

- Paracetamol (z.B. Dafalgan $\AA$, Panadol®, Acetalgin $\AA)$

- Opioide wie Codein (z.B. als Kombinationspräparat mit Paracetamol: Co-Dafalgan®, Co-Becetamol®) oder Tramadol (z.B. Tramal $\Theta$, in Kombination mit Paracetamol: Zaldiar $\Theta)$

- Metamizol (Novalgin $\AA$, Minalgin $\circledast$ )

Nicht einnehmen sollten Patienten mit chronisch-entzündlichen Darmerkrankungen unten aufgeführte Medikamente, da diese einen Schub auslösen können:

NSAR:

- Diclofenac (z.B. Voltaren $\AA$, Arthrotec $\AA$, Ecofenac $\AA$, Olfen $\AA$, Inflamac $\AA$ )

- Ibuprofen (z.B. Brufen $\AA$, Algifor $\AA$, Dolo-Spedifen $\AA$ )

- Indometacin (z.B. Indocid $B)$

- Ketorolac (z.B. Tora-Dol $\left.{ }^{\circledR}\right)$

- Acetylsalicylsäure (Aspirin $\left.{ }^{\circledR}\right)$

- Piroxicam (z.B. Felden $\AA$, Pirocam $\AA$, Pirosol $(\AA)$

- Lornoxicam (Xefo®)

- Tenoxicam (Tilcotil $\circledast$ )

- Dexibuprofen (z.B. DexOptifen®)

- Flurbiprofen (Froben $\left.{ }^{\circledR}\right)$

- Naproxen (z.B. Proxen $\circledast$, Apranax®)

- Dexketoprofen (z.B. Ketesse ${ }^{\circledR}$ )

- Mefenacid (z.B. Ponstan $\AA$, Spiralgin $\circledast$, Mephadolor $\circledast$ )

- Nimesulid (Nisulid $\circledast$, Aulin $\AA$ ) 
- Nabumeton (Balmox®)

Coxibe (COX-2-Hemmer):

- Etoricoxib (Arcoxia®)

- Celecoxib (Celebrex®) 


\section{Fragen zu Wechselwirkung von Medikamenten mit der Grunderkrankung}

Frage 1 ((Ü2))

Welches der folgenden Medikamente kann ein Asthma bronchiale exazerbieren? ((Ü2))

(Einfachauswahl, 1 richtige Antwort)
a) Amitriptylin
b) Clemastin
c) Paracetamol
d) Diclofenac
e) Enalapril

Frage 2 ((Ü2))

Welche Aussage zur analgetischen Therapie bei Patienten mit M. Crohn ist zutreffend? ((Ü2))

(Einfachauswahl, 1 richtige Antwort)
a) Paracetamol (Dafalgan $\AA$ ) ist kontraindiziert
b) Metamizol (Novalgin $\AA$ ) ist kontraindiziert
c) Celecoxib (Celebrex®) ist nicht kontraindiziert
d) Mefenaminsäure (Mefenacid®, Ponstan $\AA)$ ist kontraindiziert
e) Tramadol $\left(\right.$ Tramal $\left.{ }^{\circledR}\right)$ ist kontraindiziert

Frage 3 ((Ü2))

Wann sollten trizyklische Antidepressiva (wie Amitriptylin z.B.) vermieden werden? ((Ü2))

(Einfachauswahl, 1 richtige Antwort)
a) Neuropathische Schmerzen
b) Unbehandeltes Engwinkelglaukom
c) Schlafstörungen
d) Depression
e) Asthma bronchiale

Frage 4 ((Ü2))

Welches der folgenden Medikamente oder Medikamentenklassen ist mit QTc-Zeit-Verlängerung assoziiert? ((Ü2))

(Einfachauswahl, 1 richtige Antwort)
a) Ibuprofen
b) Coxibe
c) Paracetamol
d) Amitriptylin
e) Diclofenac

Frage 5 ((Ü2))

Welches Antihistaminikum hat klinisch bedeutsame anticholinerge Wirkungen?

(Einfachauswahl, 1 richtige Antwort)
a) Benocten $\AA$ (Diphenhydramin)
b) Telfast ${ }^{\circledR}$ (Fexofenadin)
c) Aerius $\AA$ (Desloratadin) 
d) Xyzal® (Levocetirizin)

e) Claritine $\AA_{(L o r a t a d i n)}$

\title{
Autoren ((Ü3))
}

Klinik für Klinische Pharmakologie und Toxikologie, Universitätsspital Zürich

Dr. med. A. Taegtmeyer PhD, Dr. med. I. Curkovic, Dr. med. N. Corti,

\author{
Korrespondenzadresse ((Ü2)) \\ Dr. med. Natascia Corti \\ Klinik für Klinische Pharmakologie und Toxikologie \\ Universitätsspital Zürich \\ Rämistrasse 100 \\ 8091 Zürich \\ natascia.corti@usz.ch
}




\section{Bibliographie ((Ü3))}

1. Lindblad Cl, Hanlon JT, Gross CR, Sloane RJ, Pieper CF, Hajjar ER, et al.: Clinically important drugdisease interactions and their prevalence in older adults. Clin Ther 2006; 28(8): 1133-1143.

2. Fick DM, Cooper JW, Wade WE, Waller JL, Maclean JR, Beers MH: Updating the Beers criteria for potentially inappropriate medication use in older adults: results of a US consensus panel of experts. Arch Intern Med 2003; 163(22): 2716-2724.

3. Ferrell B, Argoff CE, Epplin J, Fine P, Gloth FM, Herr K, et al.: Pharmacological management of persistent pain in older persons. J Am Geriatr Soc 2009;57(8):1331-1346.

4. Jordan K, Müller F, Schmoll HJ: [New antiemetic strategies - not only in oncology]. Internist (Berl) 2009; 50(7): 887-894.

5. Simons FE: Advances in H1-antihistamines. N Engl J Med 2004; 351(21): 2203-2217.

6. Gerretsen P, Pollock BG: Drugs with anticholinergic properties: a current perspective on use and safety. Expert Opin Drug Saf 2011; 10(5): 751-765. 
DOI 10.1024/1661-8157/a000878

((PX7_Rubrik CME-Antworten))

\section{Antworten zu Wechselwirkung von Medikamenten mit der Grunderkrankung}

Frage 1 ((Ü1))

Richtig ist Antwort d). ((Ü1))

Diclofenac (Voltaren $\AA$ ) ist ein NSAR and hemmt somit die Prostaglandinsynthese. In der Folge wird vermehrt Leukotrien gebildet. Leukotrien wirkt u.a. bronchospastisch und schleimbildend.

Die anderen Medikamente sind keine NSAR und können bei Asthma bronchiale eingesetzt werden.

Frage 2 ((Ü1))

Richtig ist Antwort d). ((Ü1))

NSAR und Coxibe können chronisch entzündliche Darmerkrankungen exazerbieren (siehe Fallbeispiel). Paracetamol ist nicht mit Exazerbation einer chronisch-entzündlichen Darmerkrankung assoziiert und ist ein alternatives Analgetikum für diese Patienten.

Frage 3 ((Ü1))

Richtig ist Antwort b). ((Ü1))

Trizyklische Antidepressiva (wie Amitriptylin) haben anticholinerge Wirkungen und können ein Engwinkelglaukom exazerbieren. Die anderen aufgeführten Krankheiten werden nicht durch Gabe eines trizyklischen Antidepressivums verschlechtert.

Frage 4 ((Ü1))

Richtig ist Antwort c). ((Ü1))

Trizyklische Antidepressiva wie Amitriptylin sind im Gegensatz zu Paracetamol, NSAR und selektiven COX-2Hemmern mit einer Verlängerung der QTc-Zeit assoziiert.

Frage 5 ((Ü1))

Richtig ist Antwort a). ((Ü1))

Benocten $\circledast$ (Diphenhydramin) hat eine starke anticholinerge Wirkung. Die anderen aufgeführten Antihistaminika gehören zu der zweiten oder dritten Generation und weisen keine klinisch bedeutsame anticholinerge Aktivität auf. 